\title{
Organización asistencial de la insuficiencia cardiaca desde la perspectiva del cardiólogo
}

\author{
Organization of care for heart failure. The cardiologist view
}

\author{
Eva González Babarro \\ Servicio de Cardiología. Complexo Hospitalario de Pontevedra
}

\begin{abstract}
Heart Failure (HF), due to its high prevalence and high morbidity and mortality, has become one of the biggest health care problems in industrialized countries. The complex management of this pathology requires adherence to the guidelines, education and changes in lifestyle, in addition to treatment of other non-cardiac aspects. Because of that, specialized units have been created, many of them wiht a multidisciplinary approach, allowing a complete care of all patient problems. For their benefits, adequately demonstrated, the creation of these units has been included in the recommendations of European Society of Cardiology.
\end{abstract}

6,3 días en los servicios de cardiología ${ }^{8}$. Por ello, toda medida que reduzca días de hospitalización será coste-efectiva.

El tratamiento farmacológico de la IC ha avanzado de forma significativa en las últimas décadas. A pesar de que diversos fármacos han demostrado una reducción de la mortalidad y de número de hospitalizaciones, continúan estando infrautilizados. Los principales registros en IC nos indican que el tratamiento en la práctica clínica se aleja de las recomendaciones, tanto en fármacos empleados como en dosis ${ }^{3,9-11}$. Se ha demostrado que la adherencia a las guías de tratamiento de la IC mejora de forma significativa el curso evolutivo de los pacientes, reduciendo los ingresos a corto plazo ${ }^{12}$. Además, los avances en terapias no farmacológicas, como la terapia de resincronización cardíaca, han demostrado también mejorar la evolución de los pacientes; la indicación correcta de este tratamiento y su control requiere conocimientos especializados ${ }^{13}$.

Otro punto importante es la infravaloración de la educación del paciente y familia sobre puntos clave como dieta, ejercicio, control de peso y TA, cambios en el estilo de vida... así como la falta de atención a aspectos no cardiólogicos concomitantes.

\section{Unidades de insuficiencia cardíaca}

La complejidad en el manejo de la insuficiencia cardíaca, su comorbilidad asociada, el incremento continuo de pacientes y el coste económico ha llevado al desarrollo de programas de insuficiencia cardíaca formados por equipos multidisciplinares. Los objetivos principales de dichos programas incluyen el diagnóstico correcto, la implementación de medidas terapéuticas, la educación de pacientes y cuidadores y por supuesto la mejoría en la supervivencia y la calidad de vida del paciente ${ }^{14}$. Objetivos desglosados en tabla 1. 
Tabla 1.0bjetivos de las unidades de IC

- Protocolización de la asistencia

» Optimización diagnóstica

» Optimización farmacológica (líneas terapéuticas y dosis recomendadas)

» Indicación de tratamientos no farmacológicos (resincronización cardíaca, desfibrilador, rehabilitación cardíaca, transplante cardíaco)

» Planificación del alta hospitalaria

» Primera visita durante los 10 primeros días tras el alta

» Plan consensuado de visitas, seguimiento telefónico, asistencia domiciliaria, telemedicina

» Accesibilidad a la asistencia sanitaria (atención a demanda del paciente)

» Detección precoz de las descompensaciones: reducción de las asistencias a los servicios de urgencias, de las hospitalizaciones y de su duración

- Abordaje multidisciplinario e integrado

- Mejora del cumplimiento terapéutico

- Valoración y asistencia de aspectos no cardiológicos (dieta, actividad sexual, entrenamiento físico, apoyo psicológico, social....)

- Fomento de la educación y autocuidado

- Mejoría de la situación funcional y de la calidad de vida

- Tratamiento de la comorbilidad

- Incremento de la supervivencia

- Control de calidad y evaluación de los resultados (clínicos, económicos y psicosociales)

Tabla 2. Modelos de unidades de IC

- Modelos de atención de IC según los miembros que la componen

» Enfermería

» Equipo médico

» Fisioterapeuta

» Farmacéutico

» Dietista

» Psicólogo

» Asistente social

- Modelos de atención de IC según su localización

» Atención hospitalaria u hospital de día

» Atención extrahospitalaria

» Asistencia domiciliaria

- Modelos de atención de IC según el tipo de intervención

» Intervención médica y educativa

» Únicamente intervención educativa y apoyo
A pesar de resultados contradictorios obtenidos en diferentes estudios (ej. Estudio $\mathrm{COACH}$ no demostró una reducción significativa en mortalidad ${ }^{15}$ ) la gran mayoría han demostrado los beneficios de dichos programas. En un metanálisis de 33 estudios randomizados se demostró reducción en la mortalidad, así como reducción en hospitalización por cualquier causa y hospitalización por $\mathrm{IC}^{16}$. Similares resultados son los obtenidos en otra revisión de 29 estudios, en los que se logró una reducción del 25\%, 26\% y 19\% en mortalidad, hospitalización por IC y hospitalización por cualquier causa, respectivamente ${ }^{17}$. Por ello, la Sociedad Europea de Cardiología recomienda que todos los pacientes con IC sean incluidos en un programa de manejo multidisciplinar para disminuir el riesgo de hospitalización por IC (clase I, nivel de evidencia A) y para reducir la mortalidad (clase II, nivel de evidencia B) ${ }^{18}$.

Sin embargo, no existe un consenso generalizado sobre cuales son los mecanismos que justifican el éxito de dichos programas. Mientras que para unos autores se debe a la adherencia del paciente al tratamiento y a la detección precoz y tratamiento de las descompensaciones ${ }^{19,20}$, para otros, se centra en una mejor aplicación práctica de la evidencia científica. Tampoco existe en la literatura consenso sobre el modelo de atención óptimo, clasificándose los modelos actuales en función de los miembros que la componen, la localización y el tipo de intervención (Tabla 2).

La definición "multidisciplinar" en la literatura existente ha sido interpretada desde diferentes perspectivas. En algunos estudios se define como equipo multidisciplinar al formado por un cardiólogo con un especialista médico (ej. Médico internista) 0 al formado por éste con un médico de atención primaria. En otros, lo constituye el formado por cardiólogo con un equipo de enfermería especializada. Sin embargo, una perspectiva más completa incluiría también la presencia de fisioterapeutas, nutricionistas, farmacéuticos, servicios sociales... ${ }^{21}$.

Pero la realidad es que cada Unidad de IC se debe adecuar a las características geográficas y logísticas de las que dispone cada área sanitaria, intentando incluir como componentes básicos un cardiólogo y enfermería especializados en IC, un coordinador asistencial entre los diferentes niveles, clínicas ambulatorias de IC y la adherencia a las Guías de Práctica Clínica ${ }^{14}$.

\section{Componentes de la unidad de insuficiencia cardíaca}

\section{Equipo médico}

El equipo médico debe estar compuesto por facultativos entrenados en el manejo de la IC que pueden proceder de distintas áreas: cardiología, medicina interna, medicina de familia y geriatría. Aunque la tendencia natural será la evolución hacia una superespecialización en el manejo de la IC, es importante mantener una formación adecuada de todos los especialistas involucrados en el proceso. 
Clásicamente ha existido cierta controversia sobre quién debe ocuparse de estos pacientes. Aunque la constitución del equipo encargado puede ser muy variable, la mayor parte de las unidades de IC se fundamentan en el trabajo de uno o varios cardiólogos con enfermería especializada, sumándose el resto de los posibles participantes según la infraestructura de cada hospital. Si bien es cierto que la IC se debe a un fracaso en el funcionamiento del corazón y por tanto que el cardiólogo tiene la responsabilidad de manejar correctamente esta patología, el que se trate de una patología médica con implicaciones en varios órganos y sistemas, el elevado número de pacientes y la comorbilidad asociada implicaría a otros especialistas en el manejo de la misma. De acuerdo a esto existen centros hospitalarios en los que los programas de IC son constituidos y dirigidos por los servicios de medicina interna y geriatría. Datos obtenidos en el año 2007 en España indican que el 91\% de la unidades depende de un servicio de cardiología y el 9\% de Medicina Interna22, 23. Lo deseable sería una participación integrada de todos, con una excelente coordinación para así lograr una verdadera intervención multidisciplinar.

Todos los componentes del equipo son importantes, con funciones específicas cada uno:

El cardiólogo debe valorar a todos los pacientes en algún momento del proceso, idealmente en la fase inicial, ya que será el encargado de realizar e integrar las diferentes pruebas complementarias (ecocardiograma, ergometría, Holter, coronariografía, ... ne necesarias en el diagnóstico y tratamiento del síndrome. Incluso, la aplicación de terapias específicas y su seguimiento posterior (terapias de resincronización, desfibriladores, transplante cardíaco,...) implica la colaboración de subespecialidades dentro de la cardiología. En cuanto a la hospitalización, existen estudios que demuestran mejores resultados en aquellos pacientes ingresados en el área de cardiología ${ }^{24,25}$, así como una estancia hospitalaria menor ${ }^{8}$. Una explicación plausible sería un perfil distinto de pacientes (más jóvenes y con menor comorbilidad).

El médico internista/geriatra es el responsable asistencial durante la hospitalización y el seguimiento tras el alta hospitalaria de la mayoría de los pacientes, así como del manejo de enfermedades no cardiovasculares. Los pacientes con IC presentan múltiples enfermedades asociadas entre la que destaca hipertensión arterial, diabetes, enfermedad pulmonar obstructiva crónica, insuficiencia renal, anemia... (hasta el 40\% de los pacientes tienen más de cinco enfermedades). Muchas de ellas participan en la etiología de la IC y desencadenan episodios de descompensación cardíaca, de los que hasta el $50 \%$ se pueden prevenir con un adecuado seguimiento e intervención precoz ${ }^{26}$. El médico de atención primaria es el responsable del diagnóstico de sospecha inicial, del seguimiento en el paciente estable, y de la prevención y diagnóstico precoz de descompensacio- nes. Los pacientes diagnosticados y seguidos por cardiología constituyen solo la punta del iceberg; el resto visitan de manera regular a su médico de familia, por lo que juegan un papel fundamental tanto en las etapas precoces como en su seguimiento27. Por esto, independientemente del modelo de unidad de IC, se debe establecer una comunicación estrecha entre el equipo de ésta y atención primaria.

El médico de urgencias realiza en muchas ocasiones la atención inicial de las descompensaciones, estableciendo además el primer diagnóstico de sospecha de una IC no conocida en muchos casos.

Por último y no por ello menos importante, en los estadíos finales de la enfermedad el médico de cuidados paliativos trata los síntomas, identifica las inquietudes y ofrece el apoyo necesario al paciente y a la familia.

\section{Enfermería}

La enfermería especializada es la piedra angular en el funcionamiento de los programas de IC, pudiendo realizar de manera autónoma la mayoría de las tareas asistenciales si estas han sido adecuadamente protocolizadas. Educan a los pacientes y cuidadores en recomendaciones dietéticas, sobre factores precipitantes y sobre la medicación. Además, identifican los síntomas y signos de descompensación cardíaca, monitorizan el cumplimiento terapéutico y en algunas unidades optimizan el tratamiento farmacológico. Otra tarea importante asignada en la mayoría de las unidades es la coordinación global de toda al asistencia prestada al paciente.

La intervención de la enfermería especializada ha demostrado que reduce los ingresos hospitalarios y que mejora el estado funcional de los pacientes ${ }^{28,29}$. Cada hospital debería alcanzar el objetivo de la Sociedad Europea de Cardiología de disponer de una enfermera especializada en IC por 100000 habitantes $^{14}$.

\section{Otros staff}

- Farmacéutico: la participación en la unidad de IC mejoraría el conocimiento del paciente sobre la medicación y sus propiedades, hecho que facilita la adherencia al tratamiento, así como también en la identificación de reacciones adversas y/o interacciones medicamentosas ${ }^{21}$.

- Dietista: el manejo dietético de los pacientes con IC es complejo, dado que suelen coexistir varios problemas nutricionales, tales como retención hidrosalina, malnutrición, obesidad, diabetes... Aunque la pérdida de peso en pacientes obesos es compleja, es más importante la ayuda del dietista en los pacientes con caquexia cardíaca.

- Fisioterapeuta/rehabilitador : El paciente con IC presenta una capacidad de ejercicio disminuida. Con la finalidad de incrementar su capacidad funcional es importante entrenar los grupos musculares necesarios para la vida diaria. Pero 
la posibilidad de inclusión de todo paciente con IC en un programa de rehabilitación cardíaca no es realista, al menos en nuestros días, dado el escaso numero de centros especializados en la misma, pudiendo ser de ayuda el establecer recomendaciones sobre tipo, duración e intensidad de ejercicios $^{21}$.

- Psicólogo: se ha demostrado que los factores psicológicos (sobre todo, ansiedad, depresión y pérdida de calidad de vida) son predictores independientes de morbimortalidad en IC . La presencia de un psicólogo puede ayudar tanto a pacientes como a familiares a hacer frente a los efectos de la IC y a educar a médicos y enfermeras en la identificación de síntomas sutiles de depresión.

- Asistente social: los servicios sociales identifican las necesidades de los pacientes, les optimizan los ayudas económicas y los recursos en domicilio con la finalidad de conseguir unos cuidados adecuados tras el alta hospitalaria.

\section{Pacientes}

Clásicamente los programas en el manejo multidisciplinar de la IC se centraron en los estadíos más avanzados de la enfermedad, obteniendo resultados positivos en calidad de vida, rehospitalizaciones y mejoría en la utilización de terapias. Sin embargo, y de acuerdo a la literatura existente hay datos que orientan hacia beneficios similares si los programas se iniciasen en estadíos más precoces de la enfermedad ${ }^{9}$. Por ello a todo paciente con IC se le debería ofertar el acceso a un programa de IC. Sin embargo, la realidad en nuestros días es que el número de pacientes atendidos en estas unidades representa sólo un pequeño porcentaje de la población total de enfermos, con un perfil clínico diferente en cada programa.

Existen unidades en las que todos los pacientes tienen menos de 65 años (pese a que la prevalencia de IC aumenta con la edad), mientras que en otras unidades uno de los criterios de entrada sería la existencia de disfunción sistólica moderadasevera, y por tanto excluiría a los pacientes con función sistólica conservada. Como se mencionó anteriormente, son los pacientes en clase funcional avanzada (NYHA III ó IV) los que más se benefician de estas unidades, por lo que se suele limitar la entrada al resto de pacientes. Podríamos concluir que cada unidad debe ser la responsable, en función de los recursos materiales y profesionales de los que disponga, de establecer el perfil de pacientes a los que incluiría en sus programas.

Por otra parte, independiente de las características de la población incluida en nuestros programas, todos ellos deberán aportar a los pacientes unos objetivos básicos que incluirían desde el conocimiento de las características de su enfermedad, su pronóstico, tratamiento, hábitos recomendados y tratamiento a seguir ( todo ello con lenguaje claro y comprensible), hasta el reconocimiento de los datos de alarma (descompensación), la reducción del número de hospitalizaciones, la mejoría de su disnea (mejora en su clase funcional NYHA) y la existencia de una vía de comunicación rápida con el personal ante duda 0 empeoramiento.

\section{Ámbito asistencial}

La unidades de IC pueden abarcar distintos ámbitos de actuación: ambulatorio, hospitalario, seguimiento telefónico, hospital de día.... todo ello dependiendo de la dimensión del equipo responsable.

La forma más sencilla de poder realizar el manejo del paciente con IC, y la de inicio de la mayoría de las unidades, es mediante una consulta especializada. El seguimiento telefónico puede servir de instrumento complementario a este modelo, sin embargo de manera aislada la consulta únicamente telefónica, como es obvio, resulta bastante limitada.

La posibilidad de utilización del hospital de día permite evitar muchas hospitalizaciones, al permitir la administración de tratamiento endovenoso en las descompensaciones incipientes, agilizar la realización de pruebas complementarias y aplicar otros tratamientos no factibles de manera ambulatoria.

Idealmente, y si la disponibilidad de recursos lo permite, se puede expandir la actuación a pacientes hospitalizados, bien ingresados en camas propias, o bien actuando como interconsultores de los pacientes ingresados en otros servicios 0 como intervención previo al alta hospitalaria. Los programas más complejos, habitualmente con mayor dotación de recursos, incluyen coordinación con atención primaria, asistencia a domicilio o incluso sistemas de telemedicina ${ }^{30}$.

\section{Proceso asistencial de insuficiencia cardíaca}

\section{(Fig. 1)}

\section{Entrada}

Los pacientes pueden ser derivados a la unidad de IC desde el ámbito hospitalario (servicio de urgencias, hospitalización, consultas externas de cardiología, medicina interna y otras especialidades) 0 desde el ámbito ambulatorio (atención primaria).

A todo paciente con síntomas y signos sospechosos de IC se le realizará, previo a su derivación, un electrocardiograma, analítica completa con péptido natriurético y radiografía de tórax ( según disponibilidad de cada centro ). Se tendría en cuenta que si no presentase ninguna alteración en alguna de las pruebas anteriores sería necesario la exclusión de otros diagnósticos previo a su derivación a la unidad de IC.

Dependiendo de la situación clínica y hemodinámica, en ocasiones, será necesario derivar al paciente al Servicio de Urgencias, donde de acuerdo a su evolución se procederá al ingreso hospitalario (en el área de Medicina Interna o Cardiología según los protocolos de cada centro hospitalario) o será remitido de manera ambulatoria a la unidad de IC. 
Figura 1.-Proceso asistencial en IC

(modificado de Standars for delivering heart failure care, European Society of Cardiology Heart Failure Association)

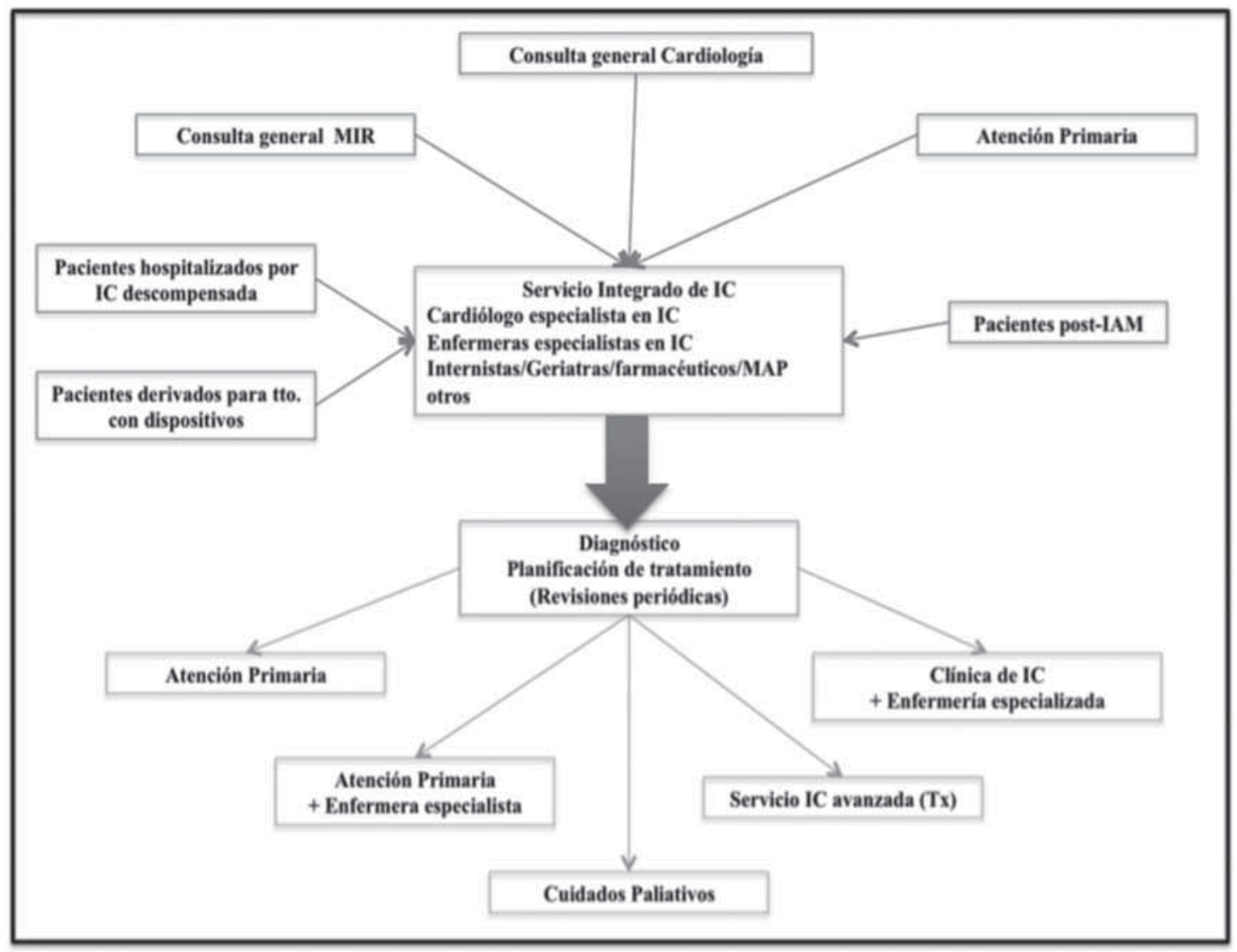

\section{Diagnóstico}

Las pruebas de imagen juegan un papel central en el diagnóstico de IC y por tanto en la orientación del tratamiento a realizar. De todas las pruebas disponibles el ecocardiograma es el método de elección en un paciente con sospecha de IC por razones de eficacia, accesibilidad, seguridad y coste, si bien puede ser complementada por otras modalidades de imagen ${ }^{31,32}$. A todo paciente con sospecha de IC se le debe realizar, además de una valoración clínica adecuada, dicha prueba, idealmente durante la primera asistencia a la unidad de IC (ejemplo: valoración por el cardiólogo de la unidad en consulta con disponibilidad de ecocardiograma).

Las guías de la Sociedad Europea de Cardiología incluyen la realización de un ecocardiograma transtorácico en IC con un grado de recomendación I, nivel de evidencia $\mathrm{C}^{18}$. Aconsejan además su realización en todo paciente con sospecha de IC y alteración en los niveles de péptidos natriuréticos y del electrocardiograma.

Se recomienda también su realización en aquellos pacientes con niveles normales de péptico natriurético pero con electro- cardiograma alterado 0 en aquellos pacientes sin anormalidad en las pruebas pero con alta probabilidad de IC (ejemplo: antecedentes de cardiopatía isquémica).

Una vez confirmado el diagnóstico de IC es necesario completar el estudio etiológico de la misma. Dado que la enfermedad coronaria es la causa de dos tercios de los pacientes con disfunción sistólica, la finalidad de la mayoría de las pruebas que complementan al ecocardiograma será la búsqueda de isquemia coronaria (ergometría, ecocardiograma de estrés, coronariografía, spect de perfusión, resonancia, tomografía. ...).

\section{Instauración de tratamiento óptimo}

Una vez realizado el diagnóstico se priorizará la planificación del tratamiento de acuerdo a las guías de práctica clínica, incluyendo las terapias médicas y no farmacológicas oportunas. Durante esta parte del proceso se iniciará también la educación del paciente y de la familia (especial énfasis en las medidas de autocuidado y adherencia al tratamiento) así como la titulación progresiva de las dosis de los diferentes fármacos. Todo ello implica la participación del equipo médico y de enfermería especializada en las consultas específicas de la unidad. 


\section{Seguimiento}

No existe un consenso sobre la periodicidad de los seguimientos. Algunas guías de práctica clínica sugieren la realización de un seguimiento semestral en aquellos pacientes estables para evaluación de los síntomas y la realización de una analítica de control que incluya una bioquímica sanguínea.

En aquellos pacientes con hospitalización reciente, durante los períodos de titulación de fármacos, tras la implantación de dispositivos o en lista de espera de transplante cardíaco se sugiere un seguimiento mucho más riguroso ${ }^{14}$.

En aquellas unidades que dispongan de sistemas de telemonitorización se podrá abordar parte del seguimiento desde esta perspectiva.

Los pacientes con tratamiento óptimo, con diagnóstico completo y con estabilidad clínica, definida ésta en un paciente con un único episodio previo como un período de 6 meses sin empeoramiento de su clase funcional, sin necesidad de hospitalización 0 asistencia a urgencias por nueva descompensación (dicho periodo se extenderá a 12 meses en aquellos pacientes que hayan presentado más de un episodio), serán remitidos para seguimiento en atención primaria.

Para ello, será necesario la realización de un informe completo y detallado, que incluya la situación cardiovascular (clase funcional), pruebas complementarias, diagnóstico etiológico, tratamiento y las instrucciones sobre los objetivos terapéuticos en los factores de riesgo cardiovascular (tensión arterial, frecuencia cardíaca, perfil lipídico y peso). El informe tendrá que especificar los criterios que puedan motivar una nueva derivación a la unidad así como una vía de contacto directo ( teléfonos de las consultas de la unidad, dirección de correo electrónico, etc), nombres del personal sanitario (médicos y enfermeras) y horario de contacto para facilitar la comunicación con el personal sanitario de atención primaria.

Existen autores que defienden la necesidad de una revisión anual en las consultas especializadas en este perfil de paciente estable. Dicha opinión se fundamenta por un lado, en que los pacientes se puedan beneficiar de los avances futuros en el manejo de esta patología, y por otro lado, en la posibilidad de revisar y reforzar las medidas de autocuidado y adherencia al tratamiento ${ }^{14}$.

Durante el seguimiento y en función de la evolución el paciente también puede ser derivado a otros servicios/unidades:

- Derivación a la Unidad de Transplante cardíaco: en aquellos pacientes con tratamiento óptimo, en mala clase funcional (definida como NYHA> II) y sin contraindicaciones absolutas para el transplante.

- Derivación a Cuidados Paliativos: pacientes en clase funcional IV de la NYHA, con hospitalizaciones frecuentes y recurrentes, sin respuesta a tratamiento médico y que no son candidatos a transplante cardíaco.

\section{Descompensaciones}

En este punto son de especial importancia las medidas de autocuidado y la identificación de los datos de alarma por parte de la paciente, así como la labor de enfermería y atención primaria para la detección precoz de las descompensaciones. Se podrá abordar las mismas de manera ambulatoria (con ajuste de medicación de administración oral), o bien en hospital de día u ingresos hospitalarios de corta estancia si precisase tratamiento endovenoso.

Superada la fase aguda, y una vez optimizado el tratamiento, el paciente podrá ser derivado nuevamente según los criterios especificados en el epígrafe de seguimiento.

\section{Conclusiones}

La unidades de IC son programas multidisciplinarios, con diversas estructuras e intervenciones, fundamentados en enfermería especializada y con unos beneficios ampliamente demostrados. Esta asistencia multidisciplinaria significa la colaboración de diversas especialidades médicas, a pesar de que, en ocasiones, el trabajo en conjunto de varios servicios médicos no sea fácil de articular.

A pesar de todos sus efectos positivos, la realidad es que el número de pacientes que se benefician de estos programas es muy limitado y que todavía no existe una generalización de estas unidades en nuestro sistema sanitario. Por eso es necesario contar con el mayor número de profesionales de la salud con especial interés en la IC y así abarcar a porcentaje mayor de pacientes con esta patología.

\section{Bibliografía}

1. Mosterd A, Hoes AW. Clinical epidemiology of heart failure. Heart 2007;93: 1137- 46.

2. Lloyd-Jones D, Adams RJ, Brown TM, Carnethon M, Dai S, De Simone G, et al. Heart disease and stroke statistics--2010 update: a report from the American Heart Association. Circulation. 2010; 121(7): 46-215.

3. Hobbs FD, Korewicki J, Cleland JG, Eastaugh J, Freemantle N. The diagnosis of heart failure in European primary care: The IMPROVEMENT Programme survey of perception and practice. Eur J Heart Fail. 2005;7(5):768-79.

4. Braunschweig F, Cowie MR, Auricchio A. What are the costs of heart failure? Europace. 2011;13:13-7.

5. Ross JS, Chen J, Lin Z, Bueno H, Curtis JP, Keenan PS et al. Recent national trends in readmission rates after heart failure hospitalization. Circ Heart Fail. 2010; 3(1):97-103.

6. Cleland JG, Swedberg K, Follath F, Komajda M, Cohen-Solal A, Aguilar JC, et al. EuroHeart Failure survey programme -- a survey on the quality of care among patients with heart failure in Europe. Part 1: patient characteristics and diagnosis. Eur Heart J. 2003; 24(5):442-63.

7. Rich MW, Beckham V, Wittenberg C, Leven CL, Freedland KE, Carney RM. A multidisciplinary intervention to prevent the readmission of elderly patients with congestive heart failure. N Engl J Med. 1995; 333(18):1190-5.

8. Navarro-López F, De Teresa E, López-Sendón JL, Castro-Beiras A. Guías del diagnóstico, clasificación y tratamiento de la insuficiencia cardíaca y del shock cardiogénico. Informe del Grupo de Trabajo de Insuficiencia Cardíaca de la Sociedad Española de Cardiología. Rev Esp Cardiol 1999; 52 (2): 1-54.

9. Nieminen MS, Brutsaert D, Dickstein K, Drexler H, Follath F, Harjola VP t al. EuroHeart Failure Survey II (EHFS II): a survey on hospitalized acute heart failure patients: description of population. Eur Heart J. 2006; 27(22):2725-36.

10. Adams KF Jr, Fonarow GC, Emerman CL, LeJemtel TH, Costanzo MR, Abraham WT et al. Characteristics and outcomes of patients hospitalized for heart failure in the United States: rationale, design, and preliminary observations from the first 100,000 cases in the Acute Decompensated Heart Failure National Registry (ADHERE). Am Heart J. 2005; 149(2): 209-16. 
11. Gheorghiade M, Abraham WT, Albert NM, Greenberg BH, O'Connor CM, She L et al. Systolic blood pressure at admission, clinical characteristics, and outcomes in patients hospitalized with acute heart failure. JAMA. 2006; 296(18): 2217-26.

12. Komajda M, Lapuerta P, Hermans N, González-Juanatey JR, Van Veldhuisen DJ, Erdmann $E$, et al. Adherente to guidelines is a predictor of outcome in chronic heart failure: the MAHLER study. Eur Heart J 2005; 26: 1653-59.

13. Bristow MR, Saxon LA, Boehmer J, Krueger S, Kass D, De Marco T, et al. Cardiacresynchronization therapy with or without an implantable defibrillator in advanced chronic heart failure. N Engl J Med 2004; 350: 2140-50.

14. McDonagh TA, Blue L, Clark AL, Dahlström U, Ekman I, Lainscak M, McDonald K, Ryder M, Strömberg A et al. European Society of Cardiology Heart Failure Association Standards for delivering heart failure care. Eur J Heart Fail. 2011; 13(3): 235-41.

15. Jaarsma T, van der Wal MH, Lesman-Leegte I, Luttik ML, Hogenhuis J, Veeger NJ et al. Effect of moderate or intensive disease management program on outcome in patients with heart failure: Coordinating Study Evaluating Outcomes of Advising and Counseling in Heart Failure (COACH). Arch Intern Med. 2008;168(3):316-24.

16. Roccaforte R, Demers C, Baldassarre F, Teo KK, Yusuf S. Effectiveness of comprehensive disease management programmes in improving clinical outcomes in heart failure patients. A meta-analysis. Eur J Heart Fail. 2005; 7(7):1133-44.

17. McAlister FA, Stewart S, Ferrua S, McMurray JJ. Multidisciplinary strategies for the management of heart failure patients at high risk for admission: a systematic review of randomized trials. J Am Coll Cardiol. 2004; 44:810-19.

18. McMurray JJ, Adamopoulos S, Anker SD, Auricchio A, Böhm M, Dickstein K et al. ESC Guidelines for the diagnosis and treatment of acute and chronic heart failure 2012: The Task Force for the Diagnosis and Treatment of Acute and Chronic Heart Failure 2012 of the European Society of Cardiology. Developed in collaboration with the Heart Failure Association (HFA) of the ESC. Eur Heart J. 2012; 33(14):1787-847.

19. Gonseth J, Guallar-Castillón P, Banegas JR, Rodríguez-Artalejo F. The effectiveness of disease management programmes in reducing hospital re-admission in older patients with heart failure: a systematic review and meta-analysis of published reports. Eur Heart J. 2004;25(18):1570-95.

20. Stewart S, Horowitz JD. Detecting early clinical deterioration in chronic heart failure patients post-acute hospitalisation-a critical component of multidisciplinary, homebased intervention? Eur J Heart Fail. 2002; 4(3):345-51.

21. Jaarsma T.Health care professionals in a heart failure team. Eur J Heart Fail. 2005 $7(3): 343-9$
22. José López-Sendón ¿Trabajo en equipo, unidades de insuficiencia cardíaca o especialidad de insuficiencia cardíaca? Rev Esp Cardiol. 2004;57:1136-8

23. Zamora E, Lupón J. Unidades de insuficiencia cardiaca en España: situación actual Rev Esp Cardiol. 2007; 60: 874-7.

24. Philbin EF, Weil HF, Erb TA, Jenkins PL. Cardiology or primary care for heart failure in the community setting: process of care and clinical outcomes. Chest 1999;116:346-54

25. Reis SE, Holubkov R, Edmundowicz D, McNamara DM, Zell KA, Detre KM etal.Treatment of patients admitted to the hospital with congestive heart failure: specialty-related disparities in practice patterns and outcomes. J Am Coll Cardiol 1997; 30: 733-38.

26. Braunstein JB, Anderson GF, Gerstenblith G, Weller W, Niefeld M, Herbert R et al. Noncardiac comorbidity increases preventable hospitalizations and mortality among Medicare beneficiaries with chronic heart failure. J Am Coll Cardiol. 2003; 42(7): 1226-33.

27. Hoes A.W., Mosterd A., Grobbee D.E. An epidemic of heart failure? Recent evidence from Europe. Eur. Heart J. 1998;19:2-9

28. Blue L, Lang E, McMurray JJ, Davie AP, McDonagh TA, Murdoch DR, Pet al. Randomised controlled trial of specialist nurse intervention in heart failure. BMJ 2001;323:715-18.

29. Stromberg A, Martensson J, Fridlund B, Levin LA, Karlsson JE, Dahlstrom U. Nurseled heart failure clinics improve survival and self-care behaviour in patients with heart failure: results from a prospective, randomised trial. Eur Heart J 2003; 24: 1014-23.

30. Bover Freire R, Ramos Isabel A, Vilacosta I. Unidades de Insuficiencia Cardíaca. En: Vilacosta I, San Román JA, Fuster V. Insuficiencia cardíaca. España: Menarini; 2010 p. 255-71.

31. Rudski LG, Lai WW, Afilalo J, Hua L, Handschumacher MD, Chandrasekaran K et al. Guidelines for the echocardiographic assessment of the right heart in adults: a report from the American Society of Echocardiography endorsed by the European Association of Echocardiography, a registered branch of the European Society of Cardiology, and the Canadian Society of Echocardiography. J Am Soc Echocardiogr. 2010; 23:685-713.

32. Sicari R, Nihoyannopoulos P, Evangelista A, Kasprzak J, Lancellotti P, Poldermans D et al. Stress echocardiography expert consensos statement: European Association of Echocardiography (EAE) (a registered branch of the ESC). Eur J Echocardiogr 2008;9: 415-37. 